\title{
Buildup of electron cloud in the PEP-II particle accelerator in the presence of a solenoid field and with different bunch pattern
}

\author{
Y. Cai* and M. Pivi ${ }^{\dagger}$ \\ Stanford Linear Accelerator Center, Stanford University, 2575 Sand Hill Road, Menlo Park, California 94025, USA \\ M. A. Furman \\ Center for Beam Physics, Accelerator and Fusion Research Division, Lawrence Berkeley National Laboratory, \\ MS 71-259, Berkeley, California 94720, USA
}

(Received 10 September 2003; published 18 February 2004)

\begin{abstract}
We have augmented the code POSINST to include solenoid fields and used it to simulate the buildup of electron cloud due in the PEP-II positron ring. We find that the distribution of electrons is strongly affected by the resonances associated with the cyclotron period and bunch spacing. In addition, we discover a threshold beyond which the electron density grows exponentially until it reaches the space charge limit. The threshold does not depend on the bunch spacing but does depend on the positron bunch population.
\end{abstract}

DOI: $10.1103 /$ PhysRevSTAB.7.024402

PACS numbers: 29.27.Bd, 79.20.Hx, 29.20.Dh

\section{INTRODUCTION}

It has been well established by many experimental evidences [1,2] at KEKB and PEP-II that the instabilities caused by electron impose a severe limitation upon the luminosity in $e^{+} e^{-}$storage rings. Based on the experiments [1] at KEKB, there exists a current threshold beyond which the vertical beam size at the interaction point starts to grow like $\sigma_{y}^{*} \propto N_{p}^{2} / S_{b}$, where $N_{p}$ is the bunch population and $S_{b}$ is the spacing between two sequential bunches. Since $N_{p}$ is normally set at the limit allowed by the beam-beam interaction, this observation implies that $S_{b}$ cannot be too small otherwise the vertical blowup degrades the luminosity. As a result, both B-factories are currently operated at $S_{b} \approx 2 \mathrm{~m}$, which is larger than its design value.

Using solenoid magnets to sweep electron cloud has been known and discussed since the design stage of the Bfactory [3]. Experimentally, the solenoid field raises the threshold of the blowup and therefore allows the increase of luminosity. On the other hand, we know from the simulation performed by Zimmermann [4] that longitudinal solenoid field $B_{s}$ confines the electrons near the wall of the vacuum chamber and therefore reduces the cloud density near the positron beam. All this indicates that both $S_{b}$ and $B_{s}$ play vital roles in the physics of electroncloud instability. In this paper, we study the dynamics between the positron beam and electron cloud with different $S_{b}$ and $B_{s}$ to reveal the physics indicated from the simulations and experiments in the PEP-II low-energy ring (LER).

\footnotetext{
*Electronic address: yunhai@slac.stanford.edu

†Electronic address: mpivi@slac.stanford.edu

‡Electronic address: mafurman@lbl.gov

Electronic address. maturnan@1bl.gov
}

\section{PHYSICAL MODEL}

\section{A. Sources of electrons}

The main sources of electrons in the PEP-II are given by residual gas ionization, photoemission from synchrotron radiation, and secondary emission from electrons hitting the walls. In the present simulations we initially generate a certain large number of electrons uniformly (in azimuth) at the chamber wall and let the electron cloud develop by the secondary emission process until an equilibrium (saturation) density is reached. This approach is valid in the limit that a small number of electrons are generated at each bunch passage compared to the equilibrium level.

\section{B. Secondary emission process}

The secondary electron yield (SEY) $\delta\left(E_{0}\right)$ and the corresponding emitted-electron energy spectrum $d \delta / d E$ $\left(E_{0}=\right.$ incident electron energy, $E=$ emitted secondary energy) are represented by a detailed model described elsewhere [5]. The parameters have been obtained from detailed fits to the measured SEY of various materials [6]. The main SEY parameters are the energy $E_{\max }$ at which $\delta\left(E_{0}\right)$ is maximum and the peak value $\delta_{\max }=\delta\left(E_{\max }\right)$; see Table I and Fig. 1. To be consistent with our previous simulations we have used a value for $\delta_{\max } \sim 2.0$ and $E_{\max }=300 \mathrm{eV}$. Furthermore, for the results shown below, we do take into account the elastic backscattered and rediffused components of the secondary emitted-electron energy spectrum $d \delta / d E$ at $E_{0} \simeq 0$. The backscattered component typically becomes more important at low incident electron energies. To account for this behavior we used a fit extrapolated data for copper measured at CERN [7] and assumed a $\delta(0) \simeq 0.5$ corresponding to a 
TABLE I. Simulation parameters for the PEP-II LER.

\begin{tabular}{ccc}
\hline \hline Parameter & Description & LER \\
\hline$E(\mathrm{Gev})$ & Beam energy & 3.1 \\
$C(\mathrm{~m})$ & Circumference & 2200 \\
$L(\mathrm{~m})$ & Length of straights & 730 \\
$N_{p}$ & Bunch population & $1.0 \times 10^{11}$ \\
$\bar{\beta}(\mathrm{m})$ & Average beta function & 17.0 \\
$\epsilon_{x, y}(\mathrm{~nm} \mathrm{rad})$ & Emittance $x, y$ & $24.0,3.0$ \\
$\sigma_{z}(\mathrm{~cm})$ & Bunch length & 1.3 \\
$S_{\mathrm{rf}}(\mathrm{m})$ & rf bucket spacing & 0.63 \\
$\delta_{\max }$ & Max secondary yield & 2.0 \\
$E_{\max }(\mathrm{eV})$ & Energy at yield max & 300 \\
$\delta(0)$ & Yield low energy & 0.5 \\
$r_{b}(\mathrm{~cm})$ & Beam pipe radius & 4.5 \\
\hline \hline
\end{tabular}

$50 \%$ reflectivity for electrons impinging the wall with an energy close to $0 \mathrm{eV}$.

\section{Simulation model}

For the purpose of these present studies we simulate the passage of a train consisting of PEP-II bunches with a bunch population of $1 \times 10^{11}$ and having a 4 or 2 bucket spacing, corresponding to 8.4 and $4.2 \mathrm{nsec}$. The stainless steel vacuum chamber is assumed to be a cylindrical perfectly conducting round pipe with a $45 \mathrm{~mm}$ radius.

Typically, the electrons are simulated by macroparticles, each one representing a defined number of electrons and carrying a fixed charge as described in [8]. The secondary electron emission mechanism adds to these a variable number of macroparticles, generated according to the SEY model mentioned above. The bunch is divided up into $N_{k}$ slices (51) and the interbunch gap into $N_{g}$ (250) intermediate steps. The image and space charge forces are

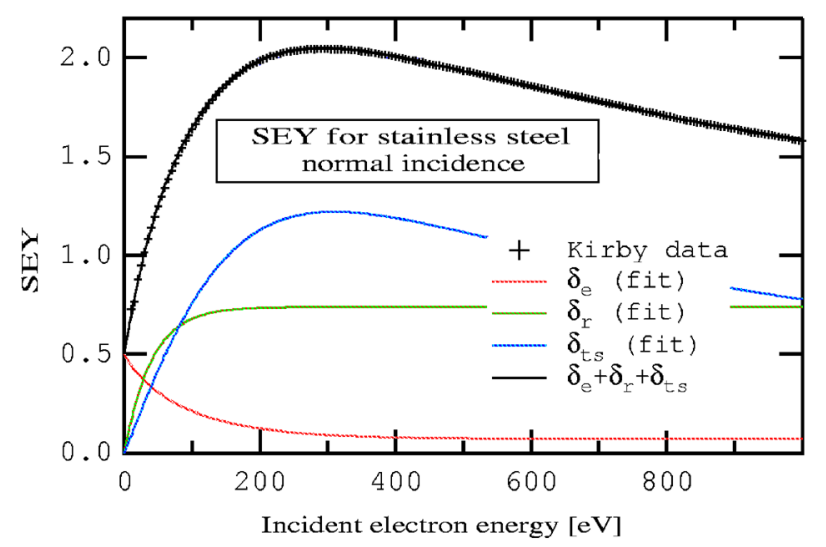

FIG. 1. (Color) The SEY for stainless steel for a SLAC standard 304 rolled sheet, chemically etched and passivated but not conditioned. Shown in the figure is the fit to the SEY experimental data and the contributions from the three physical effects of the secondary emission process, namely, the true secondary, elastic backscattered, and rediffused components. computed and applied at each slice in the bunch and each step in the gap. Typical beam and vacuum chamber parameters are listed in Table I.

For simplicity, we assume that the bunch travels at the speed of light and since the beam electric field is effectively two dimensional, it is convenient to use a complex notation to represent it $[8,9]$.

\section{Electron motion}

Our work starts with implementing longitudinal solenoid field in the code POSINST [8]. For simplicity, we assume that $\vec{B}$ is a constant and ignore any end effects of the solenoid. For a relativistic electron, the equation of motion can be written

$$
\dot{\vec{v}}=-\vec{v} \times \frac{e \vec{B}}{\gamma m c}=\vec{\omega} \times \vec{v},
$$

where $\vec{\omega}=e \vec{B} / \gamma m c$ is the cyclotron frequency of the electron. The solution of Eq. (1) is a helical orbit with the axis of the helix parallel to the magnetic field and the Larmor radius $r=v_{\perp} / \omega$. Along the field, the electron moves in a constant speed $v_{\|}$. This solution is programmed in the code to compute the motion of the electrons where the solenoid field is at presence.

The parameters used in the simulation are tabulated in Table I. $S_{b}$ has to be a multiple of the $\mathrm{rf}$ spacing $S_{\mathrm{rf}} . N_{p}$ corresponds to the value at the pezak of a typical fill in the recent operation.

\section{SIMULATION RESULTS}

\section{A. Bunch train}

Our simulation focuses on the electrons accumulated through the secondary emission from the beam pipe in the straight sections where not many primary electrons should be generated because of lack of synchrotron radiation.

The bunch pattern used in the simulation consists of a short train, a long abort gap, and a long train. The short train contains 16 bunches spaced $2 S_{\text {rf }}$, the abort gap has a length of 176 empty buckets, and the long train includes 300 bunches spaced also $2 S_{\text {rf }}$ in the simulation shown in Fig. 2. Later, when we change the bunch spacing in the simulation, the changes are always made in the long train so that the initial density of cloud is kept the same in all studies.

The initial cloud density generated by the first bunch in the short train is $1.4 \times 10^{7} \mathrm{~cm}^{-3}$ as shown in Fig. 2. The density reduces significantly in the long gap and finally builds up along the long train as seen in the figure. The final saturation level of the density does not depend upon the initial yield of electrons due to the long gap between the short and the long trains.

Without the solenoid field, the average density grows extremely fast along the train but saturates quickly near 


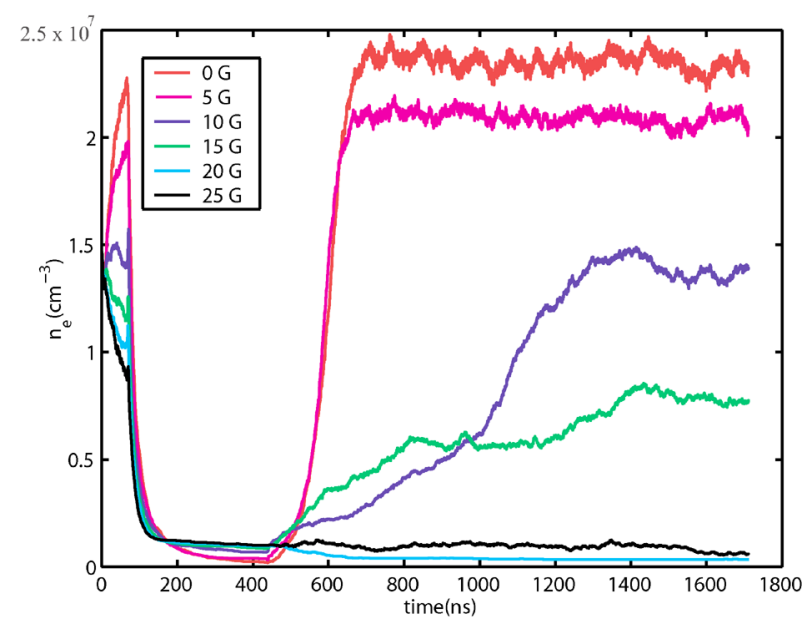

FIG. 2. (Color) Density of the electron cloud as a function of time when a bunch train passes through a stainless-steel beam pipe and longitudinal solenoid at different settings. The bunch spacing $S_{b}=2 S_{\mathrm{rf}}$.

twice the neutralization density $\rho_{e}=N_{p} / \pi r_{b}^{2} S_{b}$ due to the balance between the space charge and the secondary yield. As the solenoid field increases, both the growth rate and the equilibrium level decrease. At $B_{s}=15 \mathrm{G}$, we see a very gradual growth of the density along the train of 600 bunches. Assuming that the cloud density is proportional to the vertical beam blowup, this simulation may be used to explain the observation of the very slow blowup along the train after the initial installation of the solenoids at KEKB [1]. As $B_{s}$ reaches $25 \mathrm{G}$, the average electron density does not grow and is kept below $5 \%$ of $\rho_{e}$. That is near the density at which the head-tail instability occurs in the LER [10]. Fortunately, the density near the beam drops even more since the solenoid field restrains the electrons near the wall.

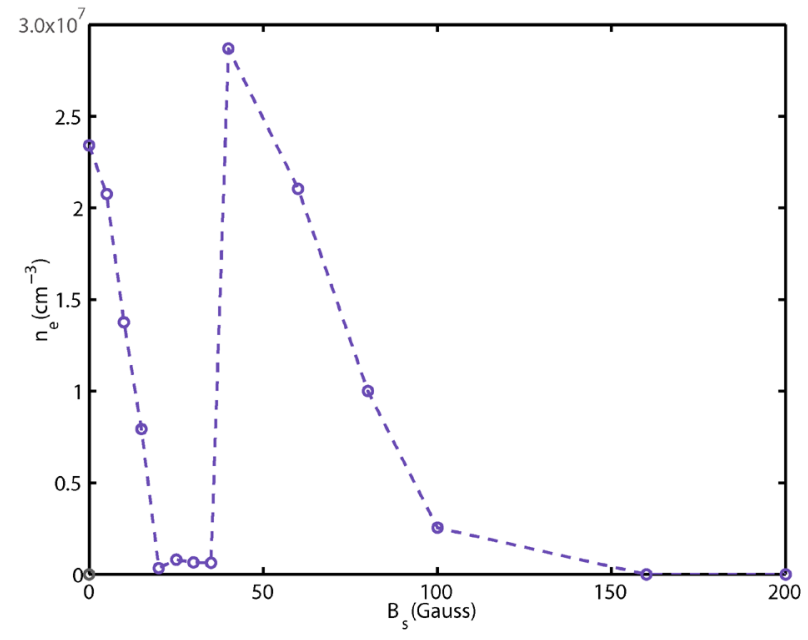

FIG. 3. (Color) Equilibrium electron-cloud density along the bunch train as a function of the solenoid field. The bunch spacing $S_{b}=2 S_{\mathrm{rf}}$.

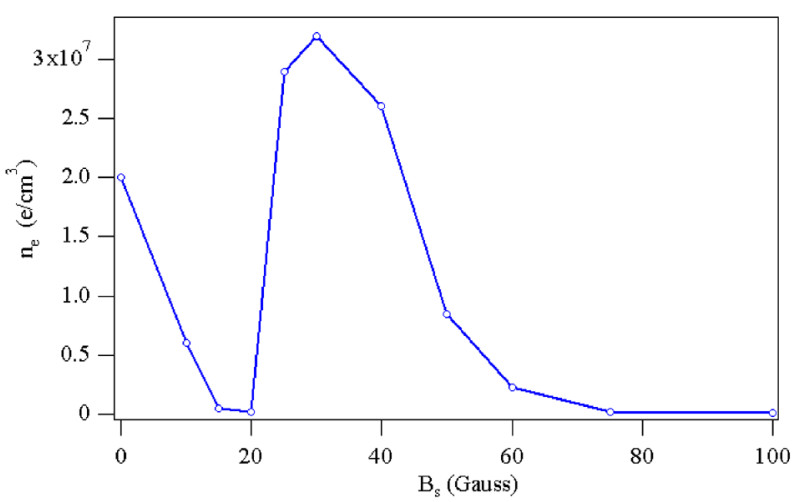

FIG. 4. (Color) Equilibrium electron-cloud density along the bunch train as a function of the solenoid field. The bunch spacing $S_{b}=3 S_{\mathrm{rf}}$.

\section{B. Cyclotron resonance}

As $B_{s}$ increases further, we find at $40 \mathrm{G}$ that the equilibrium density along the bunch train actually become larger than the density without solenoid as shown in Fig. 3 for a bunch spacing $S_{b}=2 S_{\text {rf }}$ and in Fig. 4 for a bunch spacing $S_{b}=3 S_{\mathrm{rf}}$. However, we observed that most of the electrons are confined in the vicinity of the wall as shown in Fig. 5. It is clearly seen from Figs. 3 and 4 that this phenomenon appears as a multipacting resonance.

Indeed, the result can be explained by a resonance of multipacting associated with the cyclotron frequency $\omega$ and the bunch spacing $S_{b}$. Given the low-energy nature of the secondary electrons $(100 \mathrm{eV})$, the radius of cyclotron motions is much smaller than the radius of beam pipe. The time of flight of an electron being emitted at the wall,

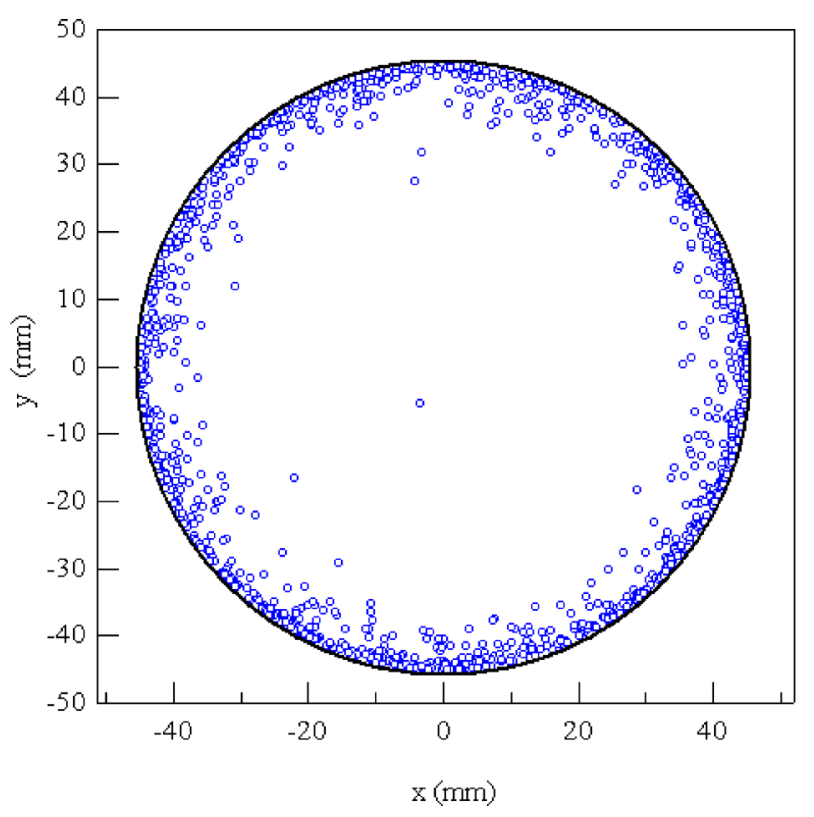

FIG. 5. (Color) Snapshot of the $x-y$ phase space for a solenoid field of $30 \mathrm{G}$, 4-rf bucket bunch spacing configuration. 
bending back by the magnetic field and finally hitting the wall, is nearly half of the cyclotron period $T_{c}=2 \pi / \omega$. The resonance occurs when the time of flight coincides with the time interval between two consecutive bunches, namely,

$$
T_{c} / 2=S_{b} / c
$$

Since $\gamma \approx 1$ for typical secondary electrons, this resonance is almost independent of the velocities of the electron and therefore much stronger than the resonance that occurred in drift space [11]. The condition of cyclotron resonance is given by

$$
B_{s}^{c}=\frac{\pi m c^{2}}{e S_{b}} .
$$

Given $S_{b}=1.26 \mathrm{~m}$, we have $B_{s}^{c}=40 \mathrm{G}$. That agrees with the simulation. In addition, we can see from Fig. 3 that a minimum density occurs at $B_{s}=B_{s}^{c} / 2$. If the bunch spacing is increased to $S_{b}=3 S_{\mathrm{rf}}, 4 S_{\mathrm{rf}}, B_{s}^{c}$ is reduced to $30 \mathrm{G}, 20 \mathrm{G}$ according to Eq. (3), as shown in Fig. 4 for the case $S_{b}=3 S_{\mathrm{rf}}$. Indeed, that is well confirmed by simulations and by a different approach using the Vlasov equation in [12]. Moreover, we find that the characters of dynamics are essentially the same if we keep the product of $S_{b}$ and $B_{s}$ as a constant.

\section{ELECTRON-CLOUD INTENSITY THRESHOLD}

It was observed at PEP-II [2] based on the pressure rise in the vacuum pumps that the multipacting of electrons in the straight sections occurs only after the total beam current exceeded a threshold. For a filling pattern of 692 bunches spaced 4-rf buckets, the multipacting threshold is about $700 \mathrm{~mA}$. Since the measurement was carried out without or with the solenoid field of $30 \mathrm{G}$, we run two simulations with the similar parameters as in the experiments in order to understand the underline mechanism of the threshold.

The results of the simulation are shown in Fig. 6. It is clearly seen from the figure that there exists a threshold beyond which the density of the electron cloud grows until it reaches equilibrium. The threshold is independent of the bunch spacing $S_{b}$ if one retains $S_{b} B_{s}$ as a constant. Above threshold, the saturated density is proportional to the line density of the beam $N_{p} / S_{b}$ indicating it is limited by the space charge. Since the peak beam current at PEPII is already operated well above the threshold, the simulation predicts a twofold increase of the electrons when the bunch spacing is shortened from $4 S_{\text {rf }}$ to $2 S_{\text {rf }}$, even if the solenoid field is doubled.

The threshold in the simulation is about $6.5 \times 10^{10}$ compared with $(4.6-6.0) \times 10^{10}$ in the observation. One should take into account that the effective peak of the secondary electron yield at the vacuum chamber

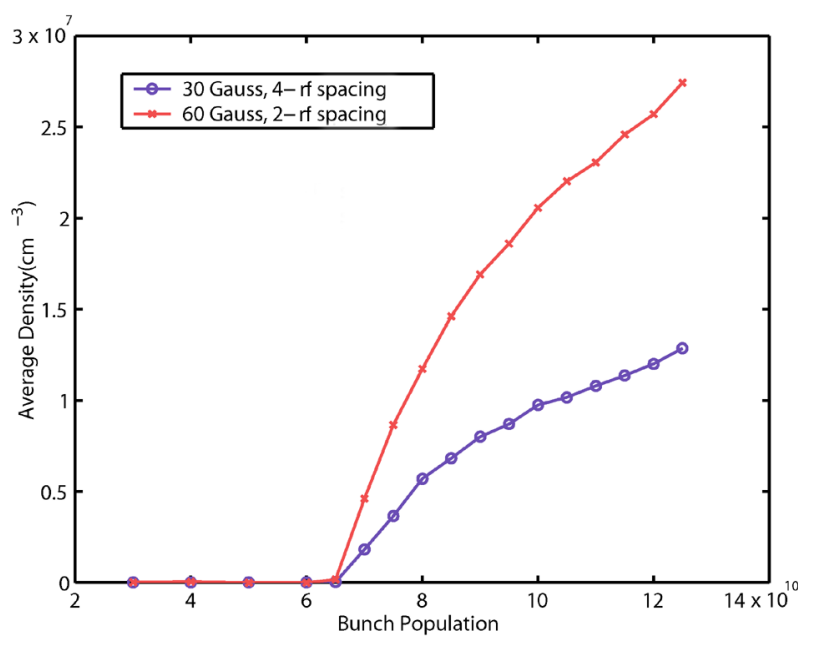

FIG. 6. (Color) Saturated density as a function of the bunch population. The circles represent the case of 4-rf spacing and a $30 \mathrm{G}$ solenoid field. The crosses represent 2 -rf spacing and a $60 \mathrm{G}$ field.

surface may be smaller than 2 as assumed in the present simulation.

A possible explanation of the intensity threshold is given by considering the details of the secondary electron yield as shown in Fig. 7. The SEY exceeds unity in our model for a $25 \mathrm{eV}$ primary electron energy. Then, inspecting Fig. 8, which shows that the average collision energy of the electrons reaches $25 \mathrm{eV}$ for a bunch population of $\sim 6.5 \times 10^{10}$, one may explain the intensity threshold as observed in Fig. 6 .

Without the solenoid, however, the simulation disagrees with the observation because of the absence of the nearby threshold in the simulation.

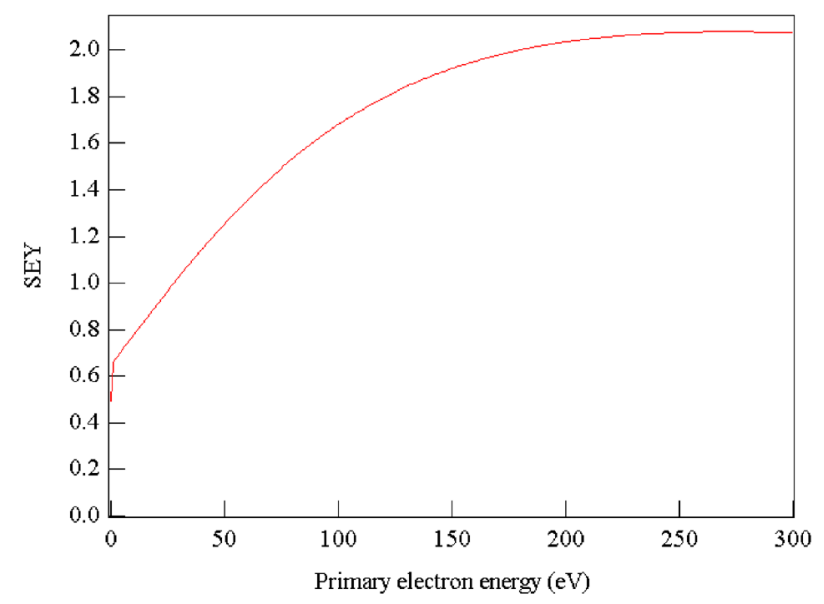

FIG. 7. (Color) Details of the secondary electron yield model used for the simulations. The secondary yield exceed 1 for a primary electron energy of $25 \mathrm{eV}$. Note that the value of the secondary electron yield close to a primary energy $0 \mathrm{eV}$ incident electron energy is $\delta(0) \sim 0.6$. 


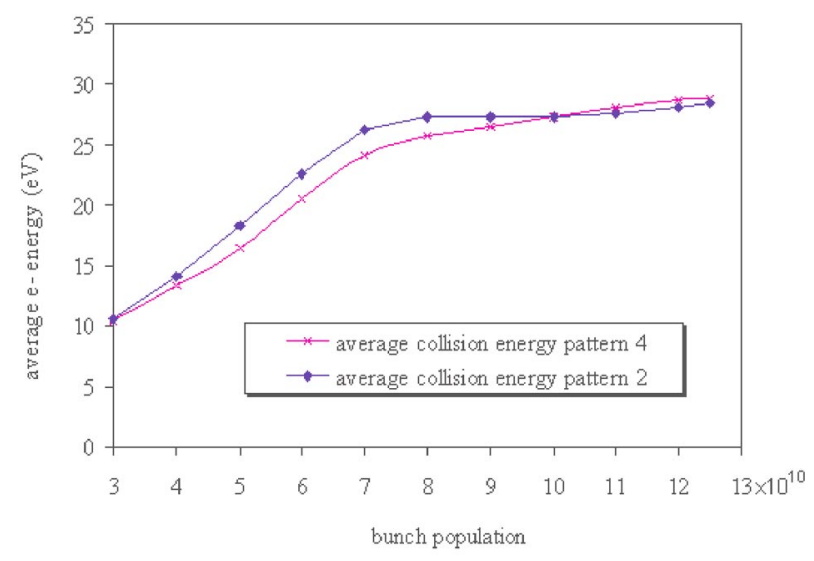

FIG. 8. (Color) Average collision energy during the whole simulation run for a different bunch population. The average energy reaches $25 \mathrm{eV}$ needed to exceed a unitary secondary yield when the bunch population is $\sim 6.5 \times 10^{10}$, explaining the intensity threshold shown in Fig. 6.

For an electron near the wall, the momentum kick due to a bunch is given by [8]

$$
\Delta p \simeq-\frac{e^{2} N_{p}}{c} \frac{2}{r_{b}},
$$

and thus the energy received from the bunch is

$$
\Delta E \simeq \frac{2 m c^{2} N_{p}^{2} r_{e}^{2}}{r_{b}^{2}},
$$

where $r_{e}$ is the classic radius of the electron. If the electron reaches the wall before the next bunch arrives $\left(B_{s}>B_{s}^{c}\right)$, multipacting of electrons occurs if

$$
\Delta E \geq E_{\delta=1},
$$

where $E_{\delta=1}$ (typically $20-100 \mathrm{eV}$ ) is the energy above which the secondary yield $\delta$ exceeds unity. This yields a threshold of bunch population

$$
N_{p}^{\mathrm{th}} \simeq \frac{r_{b}}{r_{e}} \sqrt{\frac{E_{\delta=1}}{2 E_{0}}},
$$

where $E_{0}=m c^{2}$. In this simulation, we have $E_{\delta=1} \simeq$ $30 \mathrm{eV}$. Using Eq. (7), we obtain $N_{p}^{\text {th }} \simeq 8.8 \times 10^{10}$ compared with $6.5 \times 10^{10}$ found in the simulation. Besides reducing the secondary yield, enlarging the radius of the beam pipe may be a more effective way to increase the threshold as indicated in Eq. (7).

We compute the average electron density in the vacuum beam pipe compared with the electron density within an ellipse centered on the beam axis, with and without solenoid field as shown in Fig. 9. The area of the beam ellipse is $20 \sigma_{x} \times 20 \sigma_{y}=14 \mathrm{~mm} \times 4.6 \mathrm{~mm}$. In particular, for a bunch spacing of $S_{B}=3 S_{\mathrm{rf}}$, the electron density near the beam reaches $7 \times 10^{4} \mathrm{e} / \mathrm{cm}^{3}$ when the solenoid field is set at $30 \mathrm{G}$.

\section{MULTIBUNCH INSTABILITY}

\section{A. Calculation of the vertical dipole wake field induced by the electron cloud}

For the calculation of the wake function we follow [13] as reported in [8]. After the electron cloud has reached an equilibrium density a single perturbing bunch is displaced from the central orbit by an amount $\Delta y$. In these simulations, we displace the 40th bunch vertically by $\Delta y=5 \mathrm{~mm}$. The electron cloud is perturbed

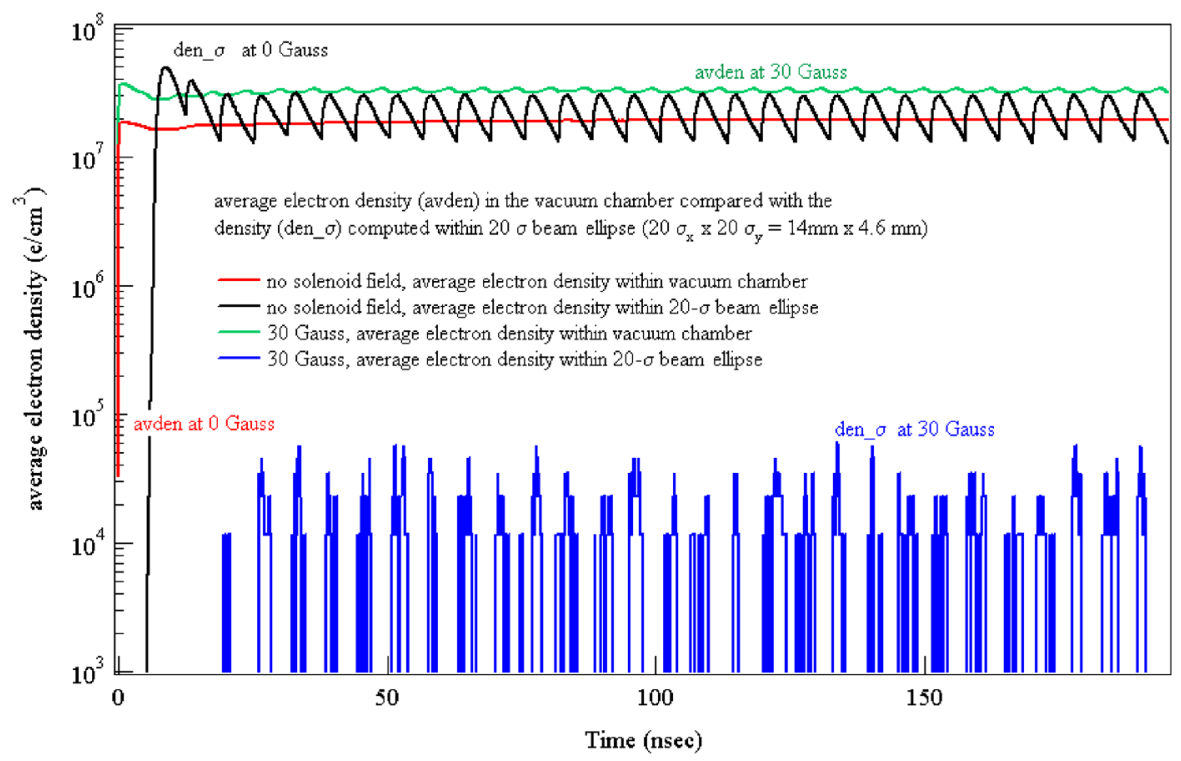

FIG. 9. (Color) Average electron density in the vacuum beam pipe compared with the electron density within an ellipse centered on the beam axis, with and without solenoid field. The area of the beam ellipse is $20 \sigma_{x} \times 20 \sigma_{y}=14 \mathrm{~mm} \times 4.6 \mathrm{~mm}$. The electron density near the beam reaches $7 \times 10^{4} e / \mathrm{cm}^{3}$ when the solenoid field is set at $30 \mathrm{G}$. 
dynamically causing a dipole wake which affects the subsequent bunches. Let the $\Delta p_{y}$ be the momentum kick experienced by the subsequent bunches as they traverse a section of length $L$. Assuming that there are $N$ sections in the ring, the dipole wake field is computed as

$$
W_{y}(z)=-\frac{N L \bar{F}_{y}}{q Q \Delta y}=-\frac{c N}{\left(e N_{p}\right)^{2}} \frac{\Delta p_{y}}{\Delta y},
$$

where $\bar{F}_{y}=\Delta p_{y} / \Delta t$ is the force acting on the subsequent bunch during the traversal of the section, $\Delta p_{y}$ is the actual momentum kick computed in the simulation, and $\Delta t=L / c$. By extracting the dipole wake function we compute the multibunch oscillation frequency in the first order approximation given in [13]. Considering the ring filled with $M$ equally spaced bunches, we compute the coherent dipole frequency $\Omega_{\mu}$ corresponding to the dipole oscillation mode $\mu$ by

$$
\Omega_{\mu}-\omega_{\beta}=\frac{c e^{2} N_{p}}{4 \pi E \nu_{\beta}} \sum_{k=0}^{n w} W\left(k s_{B}\right) e^{2 \pi i k\left(\mu+\nu_{\beta}\right) / M},
$$

where $\omega_{\beta}=\omega_{0} \nu_{\beta}$ is the betatron angular frequency, $\nu_{\beta}$ is the horizontal or vertical tune, the collective mode oscillation number is given by $\mu=0,1,2, \ldots, M-1, E$ is the beam energy, and the overall summation is extended to the wake computed for the first subsequent bunches, here $n w=10$. Since the amplitude of the oscillation for each mode $\mu$ is proportional to $\exp \left(-i \Omega_{\mu} t\right)$, the mode is unstable when $\operatorname{Im} \Omega_{\mu}$ is negative and damped when positive. Simulation results show that the wake is short range and is significant for a few trailing bunches following the perturbing bunch. Thus with good approximation, the instability growth rate $\tau_{0}^{-1}$ is given by the first $k=1$ term as

$$
\tau_{0}^{-1}=\frac{c e^{2} N_{p}}{4 \pi E \nu_{\beta}}\left|W\left(s_{B}\right)\right| .
$$

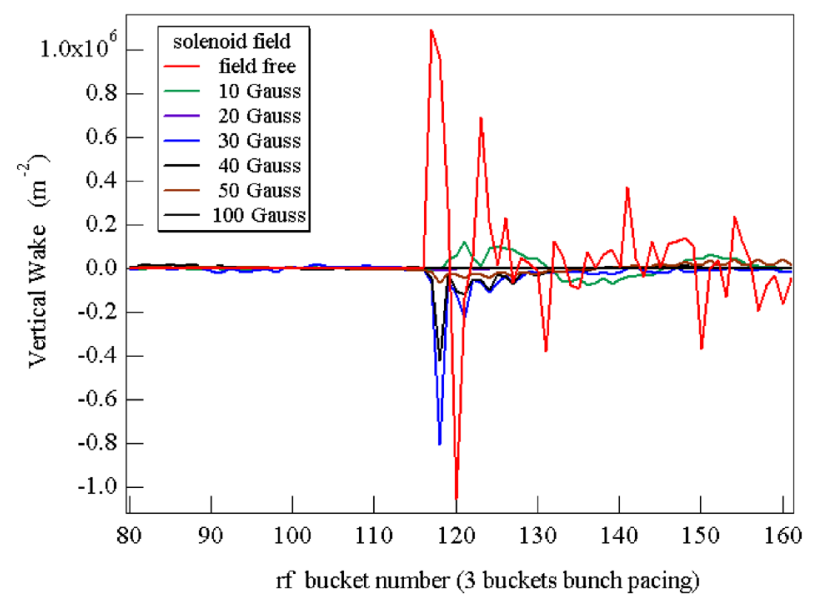

FIG. 10. (Color) Long-range wake function due to the electron cloud for a bunch spacing $S_{b}=3 S_{\mathrm{rf}}$.
TABLE II. Multibunch instability simulations. Electron cloud induced vertical tune shift $\Delta \nu_{\beta}$ and instability growth time as a function of the solenoid field for a bunch spacing $S_{b}=$ $3 S_{\mathrm{rf}}$.

\begin{tabular}{cccr}
\hline \hline Gauss & \multicolumn{1}{c}{$\Delta \nu_{\beta}$} & $\tau_{0}(\mu \mathrm{s})$ & $\tau_{0}^{-1}(1 / \mathrm{sec})$ \\
\hline No field & 0.037 & 31 & 31900 \\
10 & 0.004 & 357 & 2800 \\
20 & -0.000392 & $9.8 \times 10^{3}$ & 101 \\
30 & -0.028389 & 145 & 6885 \\
40 & -0.0369218 & 288 & 3467 \\
50 & -0.00227659 & 754 & 1325 \\
100 & $1 e-06$ & $2 \times 10^{6}$ & 0.5 \\
\hline \hline
\end{tabular}

(The growth rates as a function of the solenoid field are shown in Fig. 11 and in Table II.) We make the approximation of a constant electron density along the circumference $C$ of the ring. Finally noting that the $k=0$ mode is real and independent of the mode number $\mu$, it is interpreted as the overall coherent tune shift with the analytical expression given by

$$
\Delta \nu_{\beta}=\frac{e^{2} C N_{p}}{8 \pi^{2} E \nu_{\beta}}|W(0)| .
$$

\section{B. Effect of the solenoid field on the wake field}

As the solenoid field increases, the electrons are gradually confined within the vicinity of the wall. However, under the condition of a cyclotron resonance, there exist even more electrons than without a solenoid. Since the electrons are confined far away from the beam axis, it is not clear if these electrons could cause any instability.

To answer this question, we compute the long-range wake as a function of the solenoid field and estimate the growth rate $1 / \tau$. The wakes are shown in Fig. 10. For

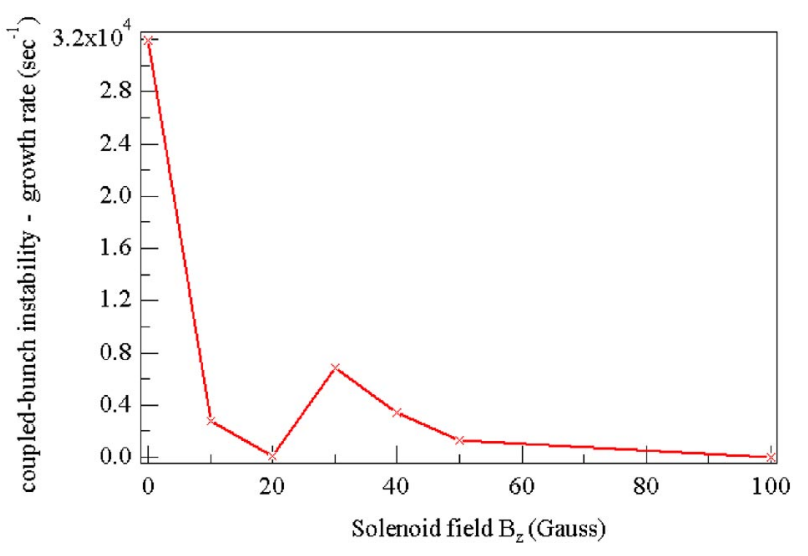

FIG. 11. (Color) Multibunch instability growth rate as a function of the solenoid field. The multipacting resonance condition at $30 \mathrm{G}$ is shown in the figure for a bunch spacing $S_{b}=3 S_{\mathrm{rf}}$. 


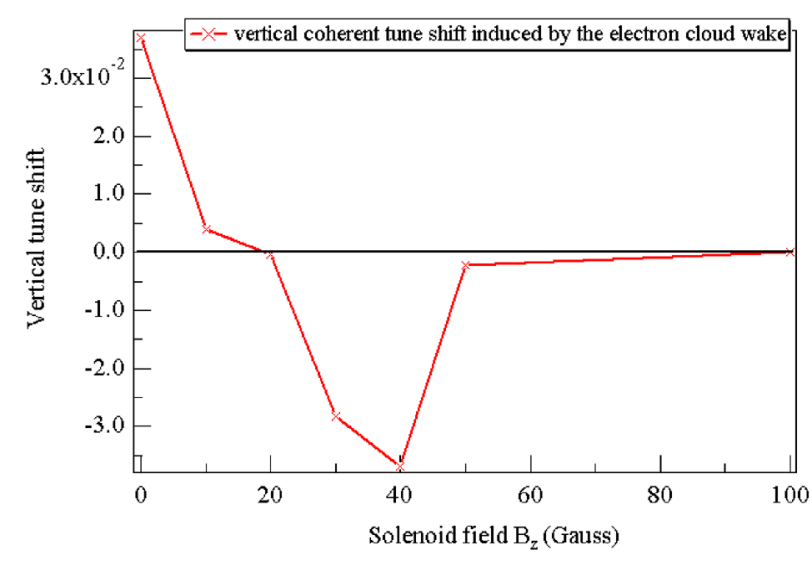

FIG. 12. (Color) Vertical tune shift induced by the electron cloud for a bunch spacing $S_{b}=3 S_{\mathrm{rf}}$.

these following simulations, we assumed a bunch spacing $S_{b}=3 S_{\text {rf }}$. Clearly from Fig. 10, the peak values of the wake are comparable at $B_{s}=0$ and $B_{s}=30 \mathrm{G}$ (at the resonance). The growth times are $\tau=31,9870$, and $145 \mu \mathrm{s}$ at $B_{s}=0,20$, and $30 \mathrm{G}$, respectively. The growth rates and the vertical tune shifts as a function of the solenoid field are shown in Figs. 11 and 12, respectively. Growth rates and tune shifts are also shown in Table II. As the solenoid field increases, one may notice that the computed long-range wake function and the vertical tune shift are changing sign, from positive to negative.

Without a solenoid field the tune shift generated by the electrons is positive because the electron cloud has a focusing effect on the positron beam. As the solenoid field increases the electrons are confined near the wall resulting in a change of sign of the tune shift similar to a conventional impedance. A neutral behavior at $20 \mathrm{G}$ is expected in the 3-rf bucket spacing.

\section{DISCUSSION}

Based on the simulation, we find that the cyclotron motion of electrons plays an important role in generating and accumulating secondary electrons. When the resonance condition is satisfied, we see a huge amount of electrons near the wall. Although they are far away from the positron beam, they still create the long-range wake strong enough to cause multibunch instability.

In addition, if the solenoid field is strong enough $B_{s}>$ $B_{s}^{c}$, we find that there exists a threshold for the electron cloud under which there is no accumulated electrons. This discovery may provide us with a method to completely eliminate the electron cloud with a large enough beam pipe and low enough secondary yield.

\section{ACKNOWLEDGMENTS}

We would like to thank Franz-Josef Decker, Stan Ecklund, Artem Kulikov, Sam Heifets, John Seeman, Mike Sullivan, and Uli Wienands for many helpful discussions. We are grateful to NERSC for supercomputer support.

[1] H. Fukuma et al., in Proceedings of the EPAC, Vienna, Austria, 2000 (CERN, Geneva, 2000), p. 1124.

[2] A. Kulikov et al., in Proceedings of the PAC01, Chicago, IL (IEEE, Piscataway, NJ, 2001), p. 1903.

[3] KEK B-factory design Report No. 95-7, 1995, Sec. 5.5.2.

[4] F. Zimmermann, CERN Report No. SL-Note-2000-004 AP, 2000.

[5] M. A. Furman and M. Pivi, Phys. Rev. ST Accel. Beams 5, 124404 (2002).

[6] R. Kirby (private communication).

[7] V. Baglin, I. Collins, B. Henrist, N. Hilleret, and G. Vorlaufer, CERN LHC Project Report No. 472, 2001.

[8] M. A. Furman and G. R. Lambertson, LBNL Note No. LBNL41123/CBP Note No. 246; SLAC Note No. PEP-II AP Note AP 97.27, 1998.

[9] M. A. Furman, Am. J. Phys. 62, 1134 (1994).

[10] Y. Cai, in Proceedings of the ECLOUD-02 Workshop (CERN, Geneva, Switzerland, 2002), p. 141.

[11] O. Gröbner, in Proceedings of the Particle Accelerator Conference (PAC 97), Vancouver, Canada (CERN No. LHC-Project-Report-127, 1997).

[12] A. Novokhatski and J. Seeman, in Proceedings of the PAC03, Portland, Oregon (IEEE, Piscataway, NJ, 2003).

[13] A. Chao, Physics of Collective Beam Instabilities in High-Energy Accelerators (John Wiley \& Sons, Inc., New York, 1993). 